\section{FRI0350 LUPUS LOW DISEASE ACTIVITY STATE (LLDAS-50) IS A SIGNIFICANT PREDICTOR FOR DAMAGE ACCRUAL AND MORTALITY: A NORWEGIAN COHORT ANALYSIS}

C. Sharma ${ }^{1}$, W. Raymond ${ }^{2}$, G. Eilertsen ${ }^{3}$, J. NOSSENT ${ }^{1} .{ }^{1}$ Rheumatology, Sir Charles Gairdner Hospital; ${ }^{2}$ University of Western Australia, Perth, Australia; ${ }^{3}$ Clinical medicine, Arctic University, Tromso, Norway

Background: Disease activity in patients with Systemic Lupus Erythematosus (SLE) is an important contributor to organ damage and premature mortality. Current indices to capture disease activity are not well suited to reflect their contribution to long term outcome. Lupus Low Disease Activity State (LLDAS) has been developed as an alternative measure of long term disease activity.

Objectives: To determine whether $50 \%$ of time spent in Lupus Low Disease Activity State (LLDAS-50) impacts on mortality and damage accrual in SLE Methods: A retrospective analysis of prospectively collected data was conducted on 3650 clinic visits by 207 patients in the Tromsø Lupus Cohort. Lupus Low Disease Activity State -50 (LLDAS50) score was defined as at least $50 \%$ of followup time with SLE Disease Activity Index (SLEDAI) $\leq 4$, no new disease activity, prednisone $\leq 7.5 \mathrm{mg} /$ day and no escalation of maintenance immunosuppressant therapy. Cox regression analysis was used to evaluate the impact of LLDAS50 in terms of mortality and damage development (either new or severe) by Systemic Lupus Erythematosus Clinical Criteria (SLICC)/American College of Rheumatology (ACR) Damage Index (SDI). New damage was defined as a rise in SDI by 1 from baseline whereas severe damage was defined as a rise of 3 points or more from baseline.

Results: The median age at diagnosis of the cohort was 34 years with the majority (84\%) being female. The median follow-up time was 125 months. A total of 69 patients $(33.5 \%$ ) spent at least half of their follow up time in LDAS, thus achieving LLDAS50. After correction for age and gender, LLDAS-50 was associated with a significant reduction in risk of having any new damage (OR $0.65 ; 95 \% \mathrm{Cl} 0.44-$ $0.96, \mathrm{p}<0.01$ ), severe damage (OR $0.46 ; 95 \% \mathrm{Cl} 0.25-0.83, \mathrm{p}<0.01$ ), and also a reduction in mortality risk (OR $0.42 ; 95 \% \mathrm{Cl} 0.21-0.82, p<0.01$ ). These values were also tested for those patients who spent $30 \%$ or more time in LDAS, and were also found to be significant for death (OR $0.46,95 \% \mathrm{Cl} .26-0.83, p<0.05)$ but not for new damage (OR $0.92,95 \% \mathrm{Cl} 0.62-1.35, \mathrm{p}=0.67$ ) or severe damage (OR $0.71,95 \% \mathrm{Cl} 0.42-1.19, \mathrm{p}=0.19$ ).

Conclusions: The significant reduction in the risk of long term damage and mortality supports the use of LLDAS50 as a therapeutic goal.

Disclosure of Interest: None declared

DOI: 10.1136/annrheumdis-2018-eular.1696

\section{FRI0351 CLINICAL CHARACTERISTICS AND OUTCOMES OF EARLY-ONSET AND LATE-ONSET LUPUS NEPHRITIS AND ITS RISK FACTORS}

C.-B. Choi, S. Won, S.-C. Bae. Rheumatology, Hanyang University Hospital for Rheumatic Diseases, Seoul, Korea, Republic Of

Background: The kidney is one of the most commonly involved major organ in systemic lupus erythematosus (SLE). It may occur as an initial presentation at the onset of SLE and it can also present later in the course of the disease.

Objectives: To compare clinical characteristics, management, and outcomes after 12 months in patients with early-onset lupus nephritis (LN) and late-onset LN and to assess the risk factors for late-onset $\mathrm{LN}$.

Methods: Patients with lupus nephritis enrolled in the Hanyang BAE Lupus Cohort were retrospectively assessed. Patients who developed LN within one year of the diagnosis of SLE (early-onset) were compared with those who developed LN more than a year later from the diagnosis of SLE (late-onset). Clinical characteristics including the features of SLE, management, and outcomes including renal responses and SLE disease activity were assessed.

Results: From 1,294 SLE patients in the Hanyang BAE Lupus Cohort, 641 (49.5\%) patients had LN. Early-onset LN was observed in 469 (73.2\%) and lateonset $L N$ in $172(26.8 \%)$. Hypertension was more frequent in early-onset $L N$ while malar rash, discoid rash, photosensitivity, oral ulcer, arthritis, leukopenia, anti-Sm $\mathrm{Ab}$, and anti-RNP Ab were more frequent in late-onset $\mathrm{LN}$. Late-onset $\mathrm{LN}$ patients also showed lower $\mathrm{C} 3$ and higher activity index in renal biopsy. There was no significant difference in ISN/RPS classification and in induction therapy. SLEDAI score at onset of LN and after 12 months was similar in the two groups. Complete and partial response rates at six months and twelve months were also similar and there were no differences in progression to end-stage renal disease or death between the two groups. Multivariate analysis identified younger age at onset, malar rash, arthritis, serositis, anti-dsDNA Ab, and anti-Sm Ab as independent risk factors for late-onset LN.
Abstract FRI0351 - Table 1. Clinical characteristics

\begin{tabular}{lccc}
\hline & $\begin{array}{c}\text { Early-onset LN } \\
(\mathrm{n}=469)\end{array}$ & $\begin{array}{c}\text { Late-onset LN } \\
(\mathrm{n}=172)\end{array}$ & $\begin{array}{c}\text { No } \mathrm{LN} \\
(\mathrm{n}=653)\end{array}$ \\
\hline Age at onset, mean \pm SD, & $23.84 \pm 10.38$ & $22.66 \pm 8.55$ & $26.73 \pm 10.51$ \\
years & & & \\
Female, $\mathrm{n}(\%)$ & $417(88.91)$ & $161(93.60)$ & $615(94.18)$ \\
Time to LN, mean $\pm S D$, & $0.06 \pm 0.19$ & $5.48 \pm 4.42$ & \\
years & & & \\
Malar rash & $200(42.64)$ & $105(61.05)$ & $262(40.12)$ \\
Discoid rash & $20(4.26)$ & $17(9.88)$ & $56(8.58)$ \\
Photosensitivity & $123(26.23)$ & $74(43.02)$ & $248(37.98)$ \\
Oral ulcer & $136(29.00)$ & $67(38.95)$ & $246(37.67)$ \\
Arthritis & $243(51.81)$ & $131(76.16)$ & $449(68.76)$ \\
Serositis & $152(32.41)$ & $46(26.74)$ & $120(18.38)$ \\
Neurologic disorder & $35(7.46)$ & $10(5.81)$ & $28(4.29)$ \\
Hematologic disorder & $383(81.66)$ & $147(85.47)$ & $539(82.54)$ \\
Immunologic disorder & $421(89.77)$ & $164(95.35)$ & $550(84.23)$ \\
ANA positivity,\% & $469(100.00)$ & $172(100.00)$ & $653(100.00)$ \\
Anti-SSa,\% & $150(33.56)$ & $69(42.59)$ & $265(41.41)$ \\
Anti-SSb,\% & $33(7.38)$ & $10(6.17)$ & $40(6.25)$ \\
Anti-RNP,\% & $130(29.08)$ & $62(38.27)$ & $219(34.27)$ \\
Antiphospholipid Ab,\% & $124(26.44)$ & $57(33.14)$ & $219(33.54)$ \\
Proteinuria, g & $2.55 \pm 2.43$ & $2.45 \pm 2.48$ & \\
SLEDAl & $6.71 \pm 4.75$ & $7.20 \pm 4.80$ & $3.98 \pm 2.86$ \\
\hline
\end{tabular}

Conclusions: Late-onset LN patients showed more mucocutaneous symptoms, autoantibodies, and higher activity index in renal biopsy compared to early-onset However, there were no differences in outcomes after 12 months. Younger age at onset, malar rash, arthritis, serositis, anti-dsDNA Ab, and anti-Sm Ab were risk factors for late-onset LN.

Disclosure of Interest: None declared

DOI: 10.1136/annrheumdis-2018-eular.3012

\section{FRI0352 DAMAGE ACCRUAL IN A LARGE MONOCENTRIC COHORT OF PRIMARY SJÖGREN'S SYNDROME PATIENTS: DETERMINANTS AND IMPACT ON PATIENT REPORTED OUTCOMES}

C. Baldini, F. Ferro, N. Luciano, E. Calabresi, E. Elefante, M. Mosca, S. Bombardieri. Rheumatology Unit, University of Pisa, Pisa, Italy

Background: Primary Sjögren's Syndrome (pSS) is a chronic progressive disease potentially leading to irreversible organ damage. To date only a limited number of studies have analysed prevalence and factors associated with damage accrual in pSS.

Objectives: a) to characterise cumulative damage in pSS patients, b) to identify determinants associated with its presence and c) to evaluate the impact of damage on patient reported outcomes (PROs).

Methods: Data from a monocentric cohort of 466 pSS patients were analysed. Glandular and extra-glandular damage manifestations were assessed by the Sjögren's Syndrome Disease Damage Index (SSDDI). Additional items of damage defined 'a priori' as being potentially related to treatment (i.e osteoporosis, diabetes, infections) were analysed separately. The EULAR Sjögren's Syndrome Disease Activity Index (ESSDAI) was used to measure disease activity at baseline and prospectively during the follow-up. The EULAR Sjogren's Syndrome Patient Reported Index (ESSPRI), Oral Health Impact Profile (OHIP) and Ocula Surface Disease Index (OSDI) were utilised to record PROs. Patients' comorbidities were assessed by the Charlson Comorbidity Index $(\mathrm{CCl})$.

Results: A total of 466 pSS patients (446 F:20 M, median age (IQR): 59 years (48-69) were included in the study. The frequency of anti-Ro-SSA in the cohor was $69.5 \%$ (324/466). The median ESSDAI was 4 (IQR 1-8) at baseline and 2 (IQR 0-5) at the last evaluation, respectively. In addition to symptomatic agents patients had been treated during the disease course with low-medium doses of glucocorticoids (GCs) (56\%), hydroxychloroquine (HCQ) (62\%) and DMARDs (16.6\%). After a median follow-up of 5 years (IQR, 2-10), 208 patients (44.6\%) had accrued some damage in either the oral damage items $(33 \%)$, ocular damage items $(20 \%)$ and/or systemic damage items (12\%). In addition, 24/466 patients had developed a non-Hodgkin lymphoma and 2 patients a multiple myeloma. The SSDDI score ranged from 0 to 14 . In the regression analysis: patients more likely to develop damage were those that were older, with a longer disease duration, higher baseline ESSDAI and who had been treated with DMARDs, whereas patients who had been ever treated with HCQ were less likely to develop diseaserelated damage. Similarly, treatment-related damage was independently associated with: disease duration, age of the patients, baseline ESSDAI, anti-Ro/SSA 